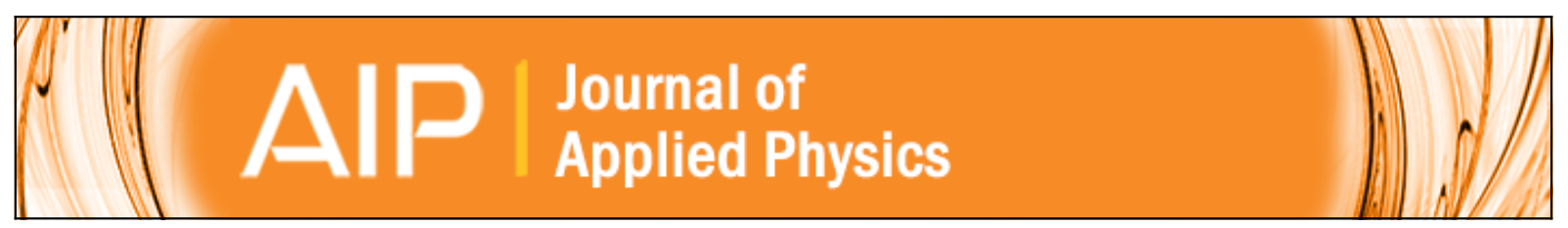

\title{
Control of the external photoluminescent quantum yield of emitters coupled to nanoantenna phased arrays
}

Ke Guo, Gabriel Lozano, Marc A. Verschuuren, and Jaime Gómez Rivas

Citation: Journal of Applied Physics 118, 073103 (2015); doi: 10.1063/1.4928616

View online: http://dx.doi.org/10.1063/1.4928616

View Table of Contents: http://scitation.aip.org/content/aip/journal/jap/118/7?ver=pdfcov

Published by the AIP Publishing

\section{Articles you may be interested in}

Remote optical sensing on the nanometer scale with a bowtie aperture nano-antenna on a fiber tip of scanning near-field optical microscopy

Appl. Phys. Lett. 106, 151104 (2015); 10.1063/1.4918531

Coupled ridge waveguide distributed feedback quantum cascade laser arrays

Appl. Phys. Lett. 106, 142104 (2015); 10.1063/1.4917294

Hybrid nanoantennas for directional emission enhancement

Appl. Phys. Lett. 105, 221109 (2014); 10.1063/1.4903219

Optical spin-to-orbital angular momentum conversion in ultra-thin metasurfaces with arbitrary topological charges Appl. Phys. Lett. 105, 101905 (2014); 10.1063/1.4895620

Ultra-sharp plasmonic resonances from monopole optical nanoantenna phased arrays Appl. Phys. Lett. 104, 231101 (2014); 10.1063/1.4881323

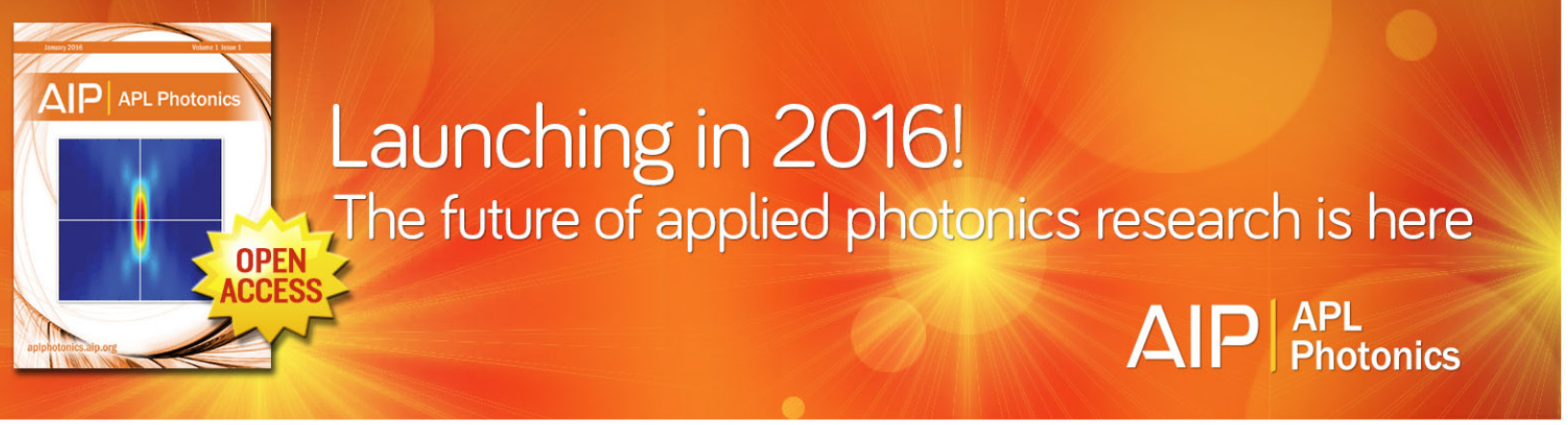




\title{
Control of the external photoluminescent quantum yield of emitters coupled to nanoantenna phased arrays
}

\author{
Ke Guo, ${ }^{1, a)}$ Gabriel Lozano, ${ }^{2, b)}$ Marc A. Verschuuren, ${ }^{1}$ and Jaime Gómez Rivas ${ }^{2,3, c)}$ \\ ${ }^{1}$ Philips Research Laboratories, High Tech Campus 4, 5656 AE Eindhoven, The Netherlands \\ ${ }^{2}$ Center for Nanophotonics, FOM Institute AMOLF, clo Philips Research Laboratories, High Tech Campus 4 , \\ 5656 AE Eindhoven, The Netherlands \\ ${ }^{3}$ COBRA Research Institute, Eindhoven University of Technology, P.O. Box 513, 5600 MB Eindhoven, \\ The Netherlands
}

(Received 1 May 2015; accepted 3 August 2015; published online 19 August 2015)

\begin{abstract}
Optical losses in metals represent the largest limitation to the external quantum yield of emitters coupled to plasmonic antennas. These losses can be at the emission wavelength, but they can be more important at shorter wavelengths, i.e., at the excitation wavelength of the emitters, where the conductivity of metals is usually lower. We present accurate measurements of the absolute external photoluminescent quantum yield of a thin layer of emitting material deposited over a periodic nanoantenna phased array. Emission and absorptance measurements of the sample are performed using a custom-made setup including an integrating sphere and variable angle excitation. The measurements reveal a strong dependence of the external quantum yield on the angle at which the optical field excites the sample. Such behavior is attributed to the coupling between far-field illumination and near-field excitation mediated by the collective resonances supported by the array. Numerical simulations confirm that the inherent losses associated with the metal can be greatly reduced by selecting an optimum angle of illumination, which boosts the light conversion efficiency in the emitting layer. This combined experimental and numerical characterization of the emission from plasmonic arrays reveals the need to carefully design the illumination to achieve the maximum external quantum yield. (C) 2015 AIP Publishing LLC.
\end{abstract}

[http://dx.doi.org/10.1063/1.4928616]

\section{INTRODUCTION}

The development of nanofabrication techniques has enabled the study of the optical properties of metallic nanostructures and the rapid growth of the field of plasmonics. Metallic nanostructures support collective electron excitations known as localized surface plasmon polaritons (LSPPs), which offer unique ways of manipulating the lightmatter interaction at the nanoscale. These characteristics have led to the term of optical antennas when referring to emitters coupled to metallic nanoparticles. ${ }^{1}$ A key quantity to evaluate the efficiency of the emission process is the intrinsic or internal photoluminescence (PL) quantum yield (IQY), which is defined as the ratio of the number of emitted photons to the number of absorbed photons by the emitter. ${ }^{2}$ IQY can be measured either relative to a fluorescent standard of known IQY or absolutely. ${ }^{3}$ Among the latter, methods based on time-resolved $\mathrm{PL}^{4,5}$ and optical power measurements ${ }^{6,7}$ have been reported. For conventional fluorescent materials, the IQY is given by the ratio of the radiative decay rate due to photon emission $\left(\gamma_{\mathrm{rad}}\right)$ to the total decay rate $\gamma_{\text {tot }}=\gamma_{\text {rad }}+\gamma_{\text {non-rad }}$, which is given by the sum of the radiative and non-radiative decay rates. ${ }^{8}$ However, for emitters coupled to resonant metallic nanostructure, the

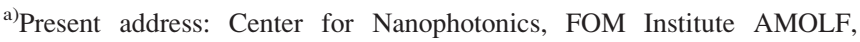
Science Park 104, 1098 XG Amsterdam, The Netherlands

${ }^{b)}$ Present address: Institute of Materials Science of Seville, Americo Vespucio 49, Seville 41092, Spain

${ }^{c)}$ Electronic mail: J.Gomez@amolf.nl
}

determination of the IQY is more challenging. The presence of LSPPs modifies the density of optical states available for the emitter to decay, providing new paths of recombination, which may increase $\gamma_{\text {rad }}{ }^{9-15}$ Meanwhile, as a limiting factor of metallic nanostructures, the Ohmic losses associated to the conductivity of the metal introduce extra non-radiative decay channels and result in an increase of $\gamma_{\text {non-rad }}{ }^{12-17}$ Since both rates $\left(\gamma_{\text {rad }}\right.$ and $\left.\gamma_{\text {non-rad }}\right)$ can be modified by the metallic nanostructures, it is difficult to separate the two contributions from the fluorescence lifetime, which is the quantity typically obtained from time-resolved PL measurements. A very important aspect, usually ignored in photoluminescence studies of plasmonic systems, is that the wave exciting the emitters can also be absorbed by the metallic nanostructures. This absorption may be dominant in the determination of the emission external quantum yield (EQY), defined as the ratio of emitted intensity to the total absorbed power by the system, due to the increase of Ohmic losses in metals at shorter wavelengths. Therefore, the IQY is insufficient for the determination of the overall efficiency of the emission and an accurate determination of the efficiency of plasmonic-based light emitting devices requires optical power measurements. In order to assess accurately the EQY, it is important to discriminate between the useful absorption by the emitter and the deleterious absorption by the metal.

In this article, we present absolute PL EQY measurements on a plasmonic-based light emitting sample using a custom-made setup based on an integrating sphere (IS). The investigated sample consists of an array of Al nanoparticles 
arranged in a square lattice with a period comparable to the wavelength of light in the visible. Over this array, a polymer layer doped with dye molecules with a high IQY is deposited. Periodic arrays of nanoparticles can work as nanoantenna phased arrays, i.e., arrays of nanoantennas in which scattered fields by the antennas interfere constructively in certain directions due to the phase delay introduced by the array. These arrays are being intensively investigated because of the narrow resonances and large field enhancements that they exhibit, ${ }^{18,19}$ which lead to a significant enhancement of the emission and a directional outcoupling of this emission in defined directions. ${ }^{20-25}$ This is a characteristic of antenna phased arrays, which beam electromagnetic radiation due to the far-field interference of the emission by the individual antennas. From the measurements, we find that although the IQY of the dye may not be significantly modified by the antenna array, the EQY has a large dependence on the angle at which the sample is excited. As we show below, this dependence is due to the fact that the illumination angle determines the way in which the optical excitation field is absorbed by the system. To unravel the connection of the EQY to the absorption of the excitation light, we employ finite element simulations to obtain the fraction of light loss by absorption in the metallic nanoparticles and the light absorbed by the dye and contributing to the emission. A careful design of the illumination conditions allows minimizing the fraction of light absorbed by the metal, and consequently increasing the light output and the EQY of the devices.

Antenna arrays provide extensive application prospects in areas such as solid-state lighting ${ }^{26-28,43}$ and lasing. ${ }^{29-31}$ The limiting factor for these applications are the absorption losses in the metal which, as we show below, can be mitigated by designing how the sample is illuminated. The reduction of these losses will also lower the threshold for lasing. Antennas arrays have also potential for photovoltaics ${ }^{32}$ and sensing applications, ${ }^{33-35}$ where absorption losses must be also reduced.

\section{FABRICATION AND CHARACTERIZATION OF THE NANOANTENNA PHASED ARRAY}

The investigated plasmonic sample is a square array of Al nanoparticles. A scanning electron microscope (SEM) image of the array is shown in Fig. 1(a) and a photograph of the sample is shown in Fig. 1(b). The array of Al nanoparticles is fabricated in a circular area with a diameter of $\simeq 5 \mathrm{~mm}$ on a fused silica glass substrate by combining substrate conformal imprint lithography with reactive ion etching. ${ }^{36,43}$ The shape of the nanoparticles is a nanopyramid with a height of $150 \mathrm{~nm}$ and a squared section with a lateral size of $120 \mathrm{~nm}$ at the top and $145 \mathrm{~nm}$ at the base. ${ }^{37}$ Over the array, a $600 \mathrm{~nm}$ thick polystyrene layer doped at $3 \%$ of mass fraction with highly efficient dye molecules (Lumogen F Red 305) is deposited by spin coating. We have determined experimentally the IQY of the dye to be $0.76 \pm 0.05$. The refractive index of such emitting layer $\left(n_{\text {dye }}=1.61\right)$ is larger than that of the substrate $\left(n_{\text {substrate }}=1.46\right)$ or the air $\left(n_{\text {air }}=1\right)$, which renders the layer of dye molecules in a waveguide. The array of nanoparticles and the emitting layer support collective resonances that are the result of the enhanced radiative coupling of LSPPs in the individual particles through diffractive orders in the plane of the array and guided modes in the emitting layer. This enhanced coupling leads to hybrid plasmonicphotonic resonances known as surface lattice resonances and quasi-guided modes, which modify the local electrical field distribution in the emitting layer and the outcoupling of its emission to free space..$^{38-41}$

The metallic nanoparticles are arranged in our sample in a square array with a lattice constant of $400 \mathrm{~nm}$ to support collective resonances that enhance the outcoupling of the red emission from the dye in the forward direction. ${ }^{27}$ To reference the photoluminescence measurements, the polymer layer containing dye molecules also covers a flat area of the substrate, i.e., without the array. The rest of the substrate is not covered with the dye layer and is sand-blasted on the back side to avoid the waveguiding of light in this area and to maximize the outcoupling efficiency of the emitted light. The roughness on the back side also helps to minimize the indirect excitation of the sample and, therefore, to suppress a common error of quantum yield measurements in an integrating sphere.

We have measured the fraction of light absorbed by the sample $\left(\mathrm{A}_{\text {tot }}\right)$ and its EQY using a blue continuous wave laser beam $\left(\lambda_{\mathrm{ex}}=448 \mathrm{~nm}\right)$, with a diameter of $\sim 1 \mathrm{~mm}$ and a power of $1.4 \mathrm{~mW}$, and an IS setup in which the illumination angle can be accurately controlled, as illustrated in Fig. 1(c). The IS is a spherical hollow cavity with an inner scattering and non-absorbing coating. Light incident on any point of (a)

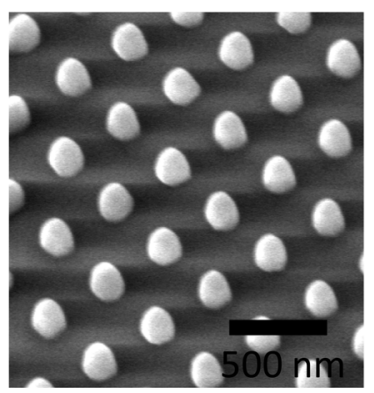

(b)

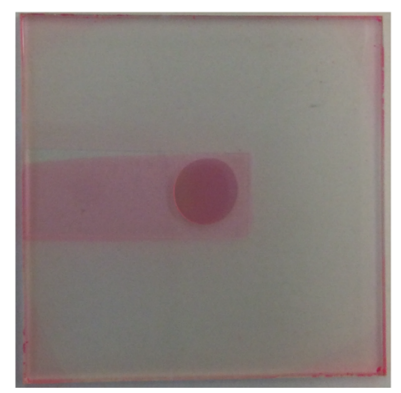

(c)

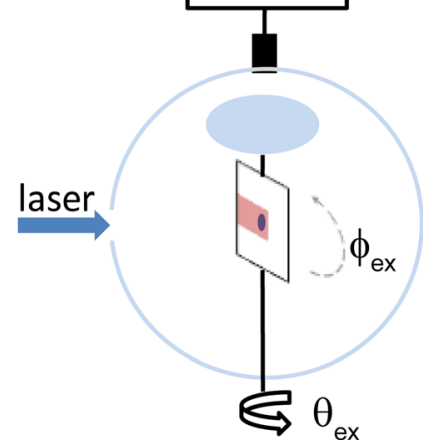

FIG. 1. (a) SEM image of a top view of the square array of $\mathrm{Al}$ nanoparticles. (b) Photograph of the sample. The dark pink circle in the center of the image is the antenna array, while the light pink area corresponds to the area covered by the dye. The glass substrate is the grey square. (c) Schematic representation of the integrating sphere setup. The sample can be rotated inside the integrating sphere while keeping the incident laser beam fixed. 
the sphere is distributed equally in all directions. A fibercoupled spectrometer is employed to collect the light scattered or emitted by the sample, which is placed in the center of the sphere. A scattering blocker is placed between the sample and the fiber of the spectrometer to prevent light from reaching directly from the sample to the detector. A small opening on the sphere allows the excitation light from the blue laser to illuminate the sample from the substrate side. In order to investigate the influence of the polar $\left(\theta_{\mathrm{ex}}\right)$ and azimuthal angle of excitation $\left(\varphi_{\mathrm{ex}}\right)$ on the EQY, the sample can be rotated using a computer-controlled stage. The fraction of light absorbed by the sample $\left(\mathrm{A}_{\text {tot }}\right)$ can be obtained experimentally as the reduction of the laser intensity when it is incident on the sample with respect to the incident intensity

$$
\mathrm{A}_{\mathrm{tot}}\left(\theta_{\mathrm{ex}}, \varphi_{\mathrm{ex}}\right)=\frac{\int_{\lambda_{\mathrm{ex}}}\left(I_{0}(\lambda)-I_{1}\left(\lambda, \theta_{\mathrm{ex}}, \varphi_{\mathrm{ex}}\right)\right) \mathrm{d} \lambda}{\int_{\lambda_{\mathrm{ex}}} I_{0}(\lambda) \mathrm{d} \lambda},
$$

where $I_{0}(\lambda)$ is the incident intensity measured with the IS and $I_{1}\left(\lambda, \theta_{\mathrm{ex}}, \varphi_{\mathrm{ex}}\right)$ is the intensity measured after impinging on the sample at the angle $\left(\theta_{\mathrm{ex}}, \varphi_{\mathrm{ex}}\right)$. The integrals are performed over the interval defined by the emission bandwidth of the laser, i.e., $\lambda_{\mathrm{ex}}=435 \mathrm{~nm}-455 \mathrm{~nm}$.

The EQY is defined as

$$
\begin{aligned}
\operatorname{EQY}\left(\theta_{\mathrm{ex}}, \varphi_{\mathrm{ex}}\right) & =\frac{I_{\mathrm{em}}\left(\theta_{\mathrm{ex}}, \varphi_{\mathrm{ex}}\right)}{A_{\mathrm{tot}}\left(\theta_{\mathrm{ex}}, \varphi_{\mathrm{ex}}\right)} \\
& =\frac{\int_{\lambda_{\mathrm{em}}} I_{1}\left(\lambda, \theta_{\mathrm{ex}}, \varphi_{\mathrm{ex}}\right) \mathrm{d} \lambda}{\int_{\lambda_{\mathrm{ex}}}\left(I_{0}(\lambda)-I_{1}\left(\lambda, \theta_{\mathrm{ex}}, \varphi_{\mathrm{ex}}\right)\right) \mathrm{d} \lambda},
\end{aligned}
$$

where $I_{\mathrm{em}}\left(\theta_{\mathrm{ex}}, \varphi_{\mathrm{ex}}\right)$ is the emission intensity normalized to the incident intensity and the integral in the numerator is

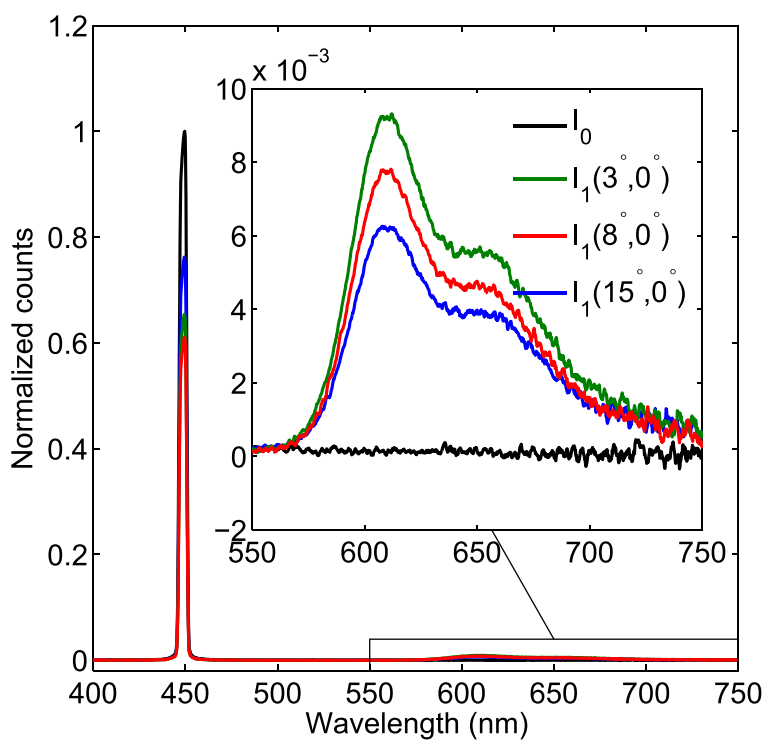

FIG. 2. Spectrum of the blue laser emission ( $I_{0}$, black curve) and spectra of the emission and the non-absorbed blue intensity $\left(I_{1}\left(\theta_{\mathrm{ex}}, \phi_{\mathrm{ex}}\right)\right)$ by the plasmonic sample shown in Fig. 1, measured at three different excitation angles, namely, $\left(\theta_{\mathrm{ex}}, \varphi_{\mathrm{ex}}\right)=\left(3^{\circ}, 0^{\circ}\right)$ green curve, $\left(8^{\circ}, 0^{\circ}\right)$ red curve and $\left(15^{\circ}, 0^{\circ}\right)$ blue curve. performed over the interval defined by the emission bandwidth of the dye molecules, i.e., $\lambda_{\mathrm{em}}=550-750 \mathrm{~nm}$.

Figure 2 shows the measured intensities for three excitation angles, namely, $\left(\theta_{\mathrm{ex}}, \varphi_{\mathrm{ex}}\right)=\left(3^{\circ}, 0^{\circ}\right),\left(8^{\circ}, 0^{\circ}\right)$, and $\left(15^{\circ}, 0^{\circ}\right)$. At the excitation wavelength $\left(\lambda_{\mathrm{ex}}=448 \mathrm{~nm}\right)$, we find $I_{1}\left(8^{\circ}, 0^{\circ}\right)<I_{1}\left(3^{\circ}, 0^{\circ}\right)<I_{1}\left(15^{\circ}, 0^{\circ}\right)$, while at the spectral range of the emission, i.e., from $550 \mathrm{~nm}$ to $750 \mathrm{~nm}$, $I_{1}\left(15^{\circ}, 0^{\circ}\right)<I_{1}\left(8^{\circ}, 0^{\circ}\right)<I_{1}\left(3^{\circ}, 0^{\circ}\right)$. In Secs. III-IV, we explain in detail the origin of the apparent absence of correlation between the total absorption of the excitation and the emission, which reveals a non-trivial dependence of the EQY with the excitation angle.

Although this manuscript focuses on the relation between the EQY and the absorption of the excitation light, an important characteristic of the sample is the emission directivity. The emission directivity of the dye layer with and without the antenna array has been measured with a Fourier microscope ${ }^{42}$ using a $100 \times$ objective with $\mathrm{NA}=0.9$. The measurement results are displayed in Fig. 3. The emission wavelength is selected using a bandpass filter at $620 \mathrm{~nm}$ with a bandwidth of $10 \mathrm{~nm}$. By imaging the back focal plane of the objective, we obtain the emission intensity as a function of emission angle $\theta_{\mathrm{em}}$ and $\varphi_{\mathrm{em}}$. The dye layer supports fundamental $(\mathrm{m}=0) \mathrm{TM}$ - and TE-waveguide modes, which are trapped in the layer and therefore cannot be detected. This leads to a low emission intensity from the dye layer as can be appreciated in Fig. 3(a). Compared with this measurement, the emission from the dye layer is enhanced by the antenna array at all directions due to the enhancement in absorption as shown in Fig. 3(b). Moreover, the antenna array couples the waveguide modes into free space through the diffractive orders $(0, \pm 1),( \pm 1$, $0)$, and $( \pm 1, \pm 1)$, resulting in a large directional emission enhancement. The dispersion of the directional enhancement follows the dispersion of the fundamental $(\mathrm{m}=0)$ TM- and TE-quasi-guided modes with diffractive order of $(0, \pm 1),( \pm 1,0)$, and $( \pm 1, \pm 1)$ as represented by black and gray lines. These guided modes have been calculated using the equations presented in Sec. III for the emission wavelength. The large enhancement in the forward emission is useful for improving the directional emission efficiency in solid state lighting applications. ${ }^{28}$
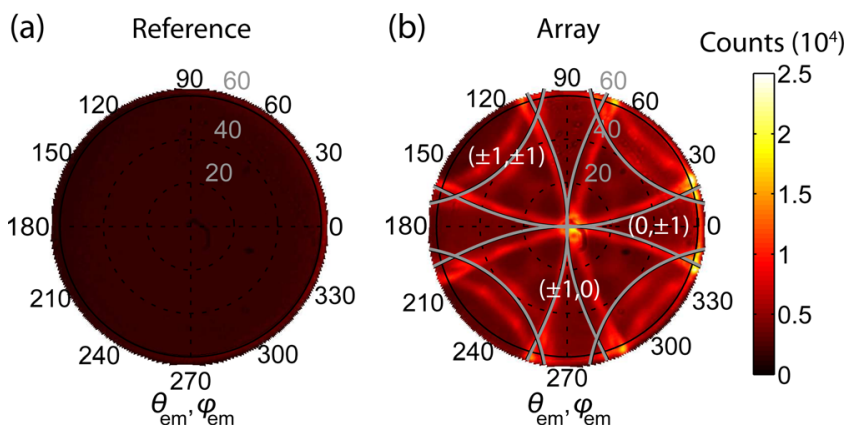

FIG. 3. Emission intensity at $\lambda=620 \mathrm{~nm}$ of (a) the dye layer on the bare substrate and (b) the dye layer on the antenna array as a function emission angle. Black curves represent the $\mathrm{TM}_{0}$-quasi-guided modes coupled to free space through the diffracted orders of $( \pm 1,0),(0, \pm 1)$ and $( \pm 1, \pm 1)$; gray curves (almost overlapping with the black curves) denote the $\mathrm{TE}_{0}$-quasiguided modes. 


\section{ANALYSIS OF THE FRACTION OF LIGHT ABSORBED BY THE NANOANTENNA PHASED ARRAY}

Numerical simulations based on a finite element method $^{43}$ are conducted to determine the EQY of the plasmonic emitting layer, which can be expressed as

$$
\mathrm{EQY}=\frac{I_{\mathrm{em}}}{A_{\mathrm{tot}}}=\frac{\mathrm{A}_{\text {dye }} \cdot \mathrm{IQY}}{\mathrm{A}_{\text {dye }}+\mathrm{A}_{\text {metal }}},
$$

where $\mathrm{A}_{\text {dye }}$ and $\mathrm{A}_{\text {metal }}$ are the fractions of the light intensity absorbed by the emitting layer and the metallic particles, respectively. It has been recently reported that the total decay rate, which defines IQY, of similar emitting layers increases only slightly in the presence of the array of metallic nanoparticles. ${ }^{27}$ Therefore, we assume that both $\gamma_{\text {rad }}$ and $\gamma_{\text {non-rad }}$ are not significantly modified by the metallic nanoparticles and we focus on the analysis of the interplay between the plasmonic array and the optical pump light. The emission of dye molecules in the proximity of the metallic nanoparticles can be quenched. However, this effect is only relevant at very short distances. ${ }^{14}$ In our samples, the dye is distributed over a broad range of distances and we expect the quenching of the emission to be negligible. Indeed, recent measurements of the decay rate of dye molecules on similar nanoparticle arrays have shown that this rate hardly change. ${ }^{27}$ This validates the approximation that the emission intensity is proportional to $A_{\text {dye }}$ and that the IQY of the dye does not change significantly by the presence of the particle array. Therefore, we focus on the analysis of the interplay between the plasmonic array and the optical pump light.

To calculate $A_{\text {dye }}$ and $A_{\text {metal }}$, we consider a unit cell of the array that contains three dielectric layers, i.e., air, the dye-doped polymer layer and a substrate, and one Al nanoparticle. We apply Floquet boundary conditions to the vertical boundaries to introduce the periodicity of the system. In order to consider the air and the substrate as semi-infinite media, perfectly matched layers on the horizontal boundaries are implemented. The different materials are characterized by their complex permittivity. We consider 2.13 as the permittivity of the fused silica substrate. The complex permittivity of the $\mathrm{Al}\left(\epsilon_{\text {metal }}\right)$ is taken from the literature, ${ }^{44}$ whereas the complex permittivity of the emitting layer $\left(\epsilon_{\text {dye }}\right)$ is obtained from ellipsometry measurements. The incident field is a plane wave propagating from the substrate with the central wavelength of the blue laser, i.e., $\lambda_{\mathrm{ex}}=448 \mathrm{~nm}$. The fraction of the incident power absorbed by the dye layer is given by

$$
\begin{aligned}
\mathrm{A}_{\text {dye }}\left(\theta_{\mathrm{ex}}, \varphi_{\mathrm{ex}}\right) & =\frac{P_{\mathrm{abs}}^{\mathrm{dye}}}{P_{0}} \\
& =\frac{1}{P_{0}} \cdot \frac{1}{2} \epsilon_{0} \operatorname{Im}\left(\epsilon_{\text {dye }}\right) \omega_{\mathrm{ex}} \int_{V_{\text {dye }}}\left|\boldsymbol{E}\left(\boldsymbol{r}, \theta_{\mathrm{ex}}, \varphi_{\mathrm{ex}}\right)\right|^{2} \mathrm{~d} \boldsymbol{r},
\end{aligned}
$$

where $P_{\mathrm{abs}}^{\mathrm{dye}}$ is the power absorbed by the dye layer, $P_{0}$ is the incident power, $\epsilon_{0}$ is the vacuum permittivity, $\operatorname{Im}\left(\epsilon_{\text {dye }}\right)$ is the imaginary component of the permittivity of the emitting layer, $\omega_{\mathrm{ex}}=2 \pi c / \lambda_{\mathrm{ex}}$ is the angular frequency at the excitation wavelength, and $\boldsymbol{E}$ is the local field for this wavelength at position $\boldsymbol{r}$. The integral is calculated over the volume occupied by the layer of dye molecules.

Analogously, $\mathrm{A}_{\text {metal }}$ is given by

$$
\begin{aligned}
\mathrm{A}_{\text {metal }}\left(\theta_{\mathrm{ex}}, \varphi_{\mathrm{ex}}\right)= & \frac{P_{\text {abs }}^{\text {metal }}}{P_{0}} \\
= & \frac{1}{P_{0}} \cdot \frac{1}{2} \epsilon_{0} \operatorname{Im}\left(\epsilon_{\text {metal }}\right) \omega_{\mathrm{ex}} \\
& \times \int_{V_{\text {metal }}}\left|\boldsymbol{E}\left(\boldsymbol{r}, \theta_{\mathrm{ex}}, \varphi_{\mathrm{ex}}\right)\right|^{2} \mathrm{~d} \boldsymbol{r},
\end{aligned}
$$

where the integral is computed over the volume occupied by the metallic nanoparticles. From Eqs. (4) and (5), it can be observed that $\mathrm{A}_{\text {dye }}$ and $\mathrm{A}_{\text {metal }}$ depend on the material permittivity at the excitation wavelength and on the spatial distribution of the electric field intensity, which in turn depends on the excitation angle.

Figures 4(a) and 4(b) show the simulated $A_{d y e}$ and $\mathrm{A}_{\text {metal }}$, respectively, as a function of $\theta_{\mathrm{ex}}$ and $\varphi_{\mathrm{ex}}$. Results are plotted in polar coordinates, being $\theta_{\mathrm{ex}}$ the radius and $\varphi_{\text {ex }}$ the polar angle. $\mathrm{A}_{\mathrm{tot}}$ and $\mathrm{A}_{\text {dye }}$ exhibit a distinct angular dependence. Figure 4(a) shows bands of high absorption in the emitting layer that are associated to hybrid plasmonicphotonic resonances supported by the array and the dye layer. ${ }^{41}$ Specifically, they correspond to the fundamental $(\mathrm{m}=0)$ TM- and TE-quasi-guided modes, i.e., when the excitation light at a particular illumination angles couples to the fundamental TM-and TE-waveguide modes of the emitting layer through diffraction with the orders $( \pm 1, \pm 1)$ of the nanoparticle array. These quasi-guided modes yield a delocalized electric near-field intensity in the emitting layer, effectively enhancing the absorption efficiency of the dye molecules. The nearly overlapping black and gray lines in Figure 4(a) represent the TM- and TE-quasi-guided modes. These modes are calculated considering the conservation of the wave vector parallel to the plane of the array due to the translational invariance of the sample in this plane and the waveguiding by total internal reflection, and are given by

$$
\pm n_{\mathrm{dye}} k_{0} \sin \theta_{\mathrm{d}} \hat{\boldsymbol{e}}_{\| \mathrm{d}}=k_{0} \sin \theta_{\mathrm{ex}}\left(\cos \varphi_{\mathrm{ex}} \hat{\boldsymbol{e}}_{x}+\sin \varphi_{\mathrm{ex}} \hat{\boldsymbol{e}}_{y}\right)+\boldsymbol{G},
$$

$$
2 k_{0} n_{\text {dye }} L \cos \theta_{\mathrm{d}}-\phi_{23}-\phi_{21}=2 m \pi,
$$

with $k_{0}=\frac{2 \pi}{\lambda_{\text {ex }}}, \theta_{\mathrm{d}}$ the angle of diffraction of the excitation light due to the array, $\hat{\boldsymbol{e}}_{\| \mathrm{d}}$ the unit vector in the plane of the array and $\boldsymbol{G}=\frac{2 \pi}{d}(p, q)$ the reciprocal lattice vector with $d$ the lattice constant and $p, q=0, \pm 1, \ldots$. In Eq. (7), $m$ is an integer that defines the order of the waveguide mode, $L$ is the thickness of the emitting layer, and $\phi_{21}$ and $\phi_{23}$ are the phase shifts due to total internal reflection. These phase shifts can be calculated for TE modes using ${ }^{45}$

$$
\begin{aligned}
& \tan \frac{\phi_{21}}{2}=\left(n_{\text {dye }}^{2} \sin ^{2} \theta_{\mathrm{d}}-n_{\text {air }}^{2}\right)^{1 / 2} /\left(n_{\text {dye }} \cos \theta_{\mathrm{d}}\right), \\
& \tan \frac{\phi_{23}}{2}=\left(n_{\text {dye }}^{2} \sin ^{2} \theta_{\mathrm{d}}-n_{\text {substrate }}^{2}\right)^{1 / 2} /\left(n_{\text {dye }} \cos \theta_{\mathrm{d}}\right),
\end{aligned}
$$

and for TM modes using 
(a) Absorption in the dye layer

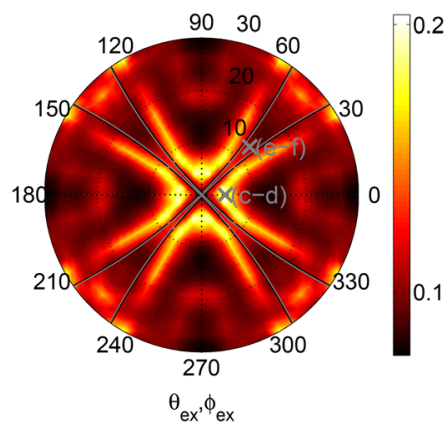

(b)

Absorption in the metal (c)

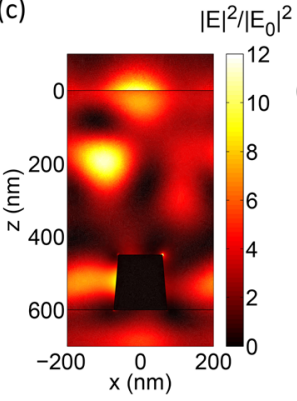

(d)

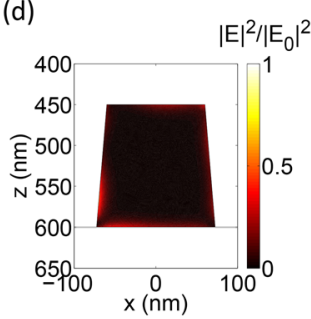

(e)

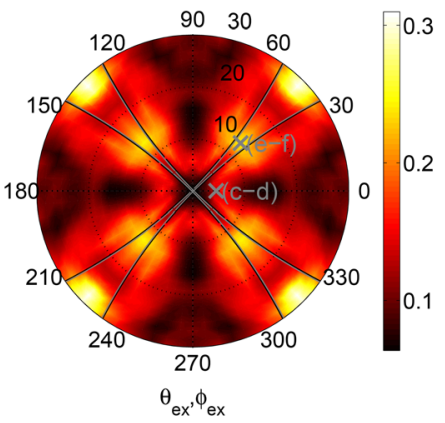

FIG. 4. Simulated (a) $\mathrm{A}_{\text {dye }}$ and (b) $\mathrm{A}_{\text {metal }}$ in an unit cell of a square array of $\mathrm{Al}$ nanoparticles with a lattice constant of $400 \mathrm{~nm}$ on a substrate with a permittivity of 2.13 , covered by a $600 \mathrm{~nm}$-thick layer with a permittivity of $2.59+0.01 \mathrm{i}$ corresponding to the dye layer. The simulations consider a p-polarized plane wave incident onto the array from the substrate with a wavelength of $\lambda_{e x}=448 \mathrm{~nm}$ at an angle $\left(\theta_{\mathrm{ex}}, \varphi_{\mathrm{ex}}\right)$. Black curves represent the $\mathrm{TM}_{0}$-quasi-guided modes coupled from free space through the diffracted orders of $( \pm 1, \pm 1)$; gray curves (almost overlapping with the black curves) denote the $\mathrm{TE}_{0}$-quasi-guided modes. (c)-(f) Simulated spatial distribution of the total electrical field intensity $\left(|E|^{2}\right)$ normalized to the incident field intensity $\left(\left|E_{0}^{2}\right|\right)$ at the $y=0$ plane in a unit cell of the array. The simulations consider a p-polarized plane wave incident at (c) and (d) $\theta_{\mathrm{ex}}=4.5^{\circ}$ and $\varphi_{\mathrm{ex}}=0^{\circ}$, and at (e) and (f) $\theta_{\mathrm{ex}}=13^{\circ}$ and $\varphi_{\mathrm{ex}}=45^{\circ}$. (d) and (f) A detail of the electric field intensity of (c) and (e), respectively, inside the Al particle.

$\tan \frac{\phi_{21}}{2}=n_{\text {dye }}^{2}\left(n_{\text {dye }}^{2} \sin ^{2} \theta_{\mathrm{d}}-n_{\text {air }}^{2}\right)^{1 / 2} /\left(n_{\text {air }}^{2} n_{\text {dye }} \cos \theta_{\mathrm{d}}\right)$,

$\tan \frac{\phi_{23}}{2}=n_{\text {dye }}^{2}\left(n_{\text {dye }}^{2} \sin ^{2} \theta_{\mathrm{d}}-n_{\text {substrate }}^{2}\right)^{1 / 2} /\left(n_{\text {substrate }}^{2} n_{\text {dye }} \cos \theta_{\mathrm{d}}\right)$.

Figures 4(c)-4(f) display numerical simulations of the local field intensity enhancement (relative to the incident field) for a plane wave incident at two different angles. The field intensity enhancement is shown in a plane intersecting the nanoparticles at their central position in a unit cell of the array. These simulations reveal that the complex photonic environment forming the sample gives rise to an inhomogeneous spatial distribution of the local field intensities around the metallic nanoparticles. To illustrate this effect, Figs. 4(c) and 4(d) and Figs. 4(e) and 4(f) display the field intensity associated to two different illumination angles: $\left(\theta_{\mathrm{ex}}, \varphi_{\mathrm{ex}}\right)$ $=\left(4.5^{\circ}, 0^{\circ}\right)$ and $\left(\theta_{\mathrm{ex}}, \varphi_{\mathrm{ex}}\right)=\left(13^{\circ}, 45^{\circ}\right)$, respectively. These angles are marked by the crosses in Figs. 4(a) and 4(b). Figure 4(c) shows a large intensity enhancement in the dye layer for $\theta_{\mathrm{ex}}=4.5^{\circ}$ and $\varphi_{\mathrm{ex}}=0^{\circ}$ as a result of the efficient coupling of the pump field to the quasi-guided mode supported by the array. In contrast, when the same structure is illuminated at $\theta_{\mathrm{ex}}=13^{\circ}$ and $\varphi_{\mathrm{ex}}=45^{\circ}$, the electric field intensity is reduced in the dye layer, whereas it is significantly larger inside the metal particle (Fig. 4(f)) compared with the field in the particle shown in Figure 4(d). We attribute this effect to the excitation of LSPPs in the individual metallic nanoparticles. $^{46}$ The electric field generated by localized resonances is confined to the edges of the nanoparticles, resulting in large $A_{\text {metal }}$.

Two azimuthal angles have been chosen to further illustrate how light is absorbed by the dye layer and the nanoantenna phased array as a function of the angle of incidence. Figures 5(a) and 5(b) show the measured $A_{\text {tot }}$ as a function of $\theta_{\mathrm{ex}}$ at $\varphi_{\mathrm{ex}}=0^{\circ}$ and $\varphi_{\mathrm{ex}}=45^{\circ}$, respectively. The corresponding simulations are shown in Figs. 5(c) and 5(d). A good agreement is found between measurements and simulations. These figures illustrate the strong dependence of the absorption with the angle of incidence. The absorbance can change from less than $20 \%$ to over $40 \%$ by varying the angle of

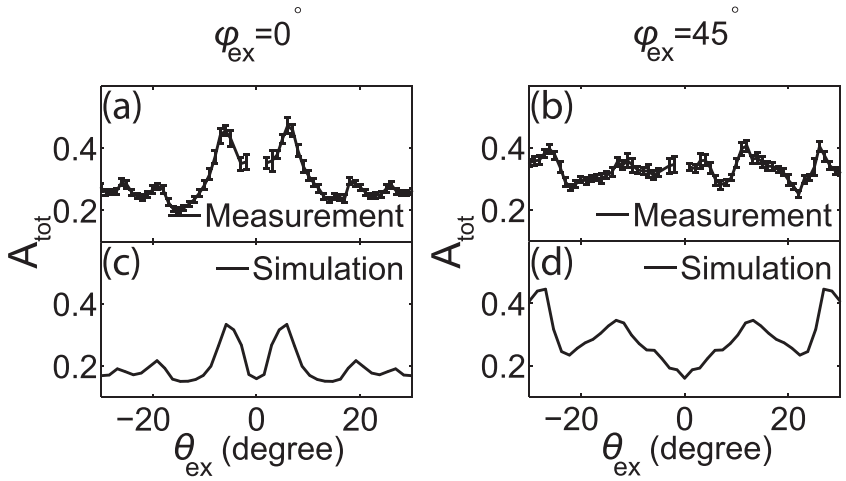

FIG. 5. (a) and (b) Measured and (c) and (d) simulated $A_{\text {tot }}$ as a function of $\theta_{\mathrm{ex}}$ for (a) and (c) $\varphi_{\mathrm{ex}}=0^{\circ}$ and (b) and (d) $\varphi_{\mathrm{ex}}=45^{\circ}$ for a square array of Al nanoparticles with a lattice constant of $400 \mathrm{~nm}$, covered by a $600 \mathrm{~nm}$ thick layer of polymer doped with dye molecules. 
incidence by less than $10^{\circ}$ (Fig. 5(a)). We attribute the large $\mathrm{A}_{\text {tot }}$ at $\theta_{\mathrm{ex}}=4.5^{\circ}$ and $\varphi_{\mathrm{ex}}=0^{\circ}$ to an increased absorption in the dye layer resulting from the coupling of the incident light to quasi-guided modes, and the peaks of $\mathrm{A}_{\text {tot }}$ observed at $\left(\theta_{\mathrm{ex}}, \varphi_{\mathrm{ex}}\right)=\left(13^{\circ}, 45^{\circ}\right)$ and $\left(\theta_{\mathrm{ex}}, \varphi_{\mathrm{ex}}\right)=\left(27^{\circ}, 45^{\circ}\right)$ to an enhanced absorption in the metal due to the excitation of LSPPs. The most important conclusion of these results is that it is possible to engineer the illumination of the plasmonicbased emitting layer such that the deleterious losses associated to the presence of the metal are minimized.

\section{ABSOLUTE DETERMINATION OF THE EXTERNAL QUANTUM YIELD OF THE NANOANTENNA PHASED ARRAY}

Figures 6(a) and 6(b) display the measurements of the absolute EQY of the sample for $\varphi_{\mathrm{ex}}=0^{\circ}$ and $\varphi_{\mathrm{ex}}=45^{\circ}$. The measurements show a fluctuating behavior with the illumination angle that originates from the dependence of $A_{\text {dye }}$ and $\mathrm{A}_{\text {metal }}$ with the angle of incidence. While $\mathrm{A}_{\text {dye }}$ determines the absorption in the layer of dye, which is proportional to the emitted intensity, the energy of the light absorbed by the metal is dissipated and does not contribute to the emission. As a consequence, $\mathrm{A}_{\text {metal }}$ reduces the $\mathrm{EQY}$ of the system.

In order to establish a direct relation between the measured EQY and the simulated absorption, we compute the EQY according to Eq. (3) with $\mathrm{A}_{\text {dye }}$ and $\mathrm{A}_{\text {metal }}$ obtained from the numerical simulations shown in Fig. 4 and $\mathrm{IQY}=0.76$ obtained from measurements. Figures 6(c) and 6(d) show the calculation results, which are in good agreement with the measurements, both in angular behavior and magnitude. For $\varphi_{\mathrm{ex}}=0^{\circ}, \mathrm{A}_{\text {dye }}$ and $\mathrm{A}_{\text {metal }}$ have similar dependencies with the angle of illumination as shown in Figs. 2(a) and 2(b). As a result, the variation in the EQY is relatively small, although two pronounced peaks in $A_{\text {tot }}$ are observed. For $\varphi_{\mathrm{ex}}=45^{\circ}$, the variation in $\mathrm{A}_{\mathrm{tot}}$ and in the EQY originate mainly from the large variation of $A_{\text {metal }}$ while $A_{\text {dye }}$ is relatively constant. Therefore, a low EQY is observed while $A_{\text {tot }}$ is large. It can be also observed that the averaged EQY decreases from EQY $\sim 0.4$ at $\varphi_{\mathrm{ex}}=0^{\circ}$ to
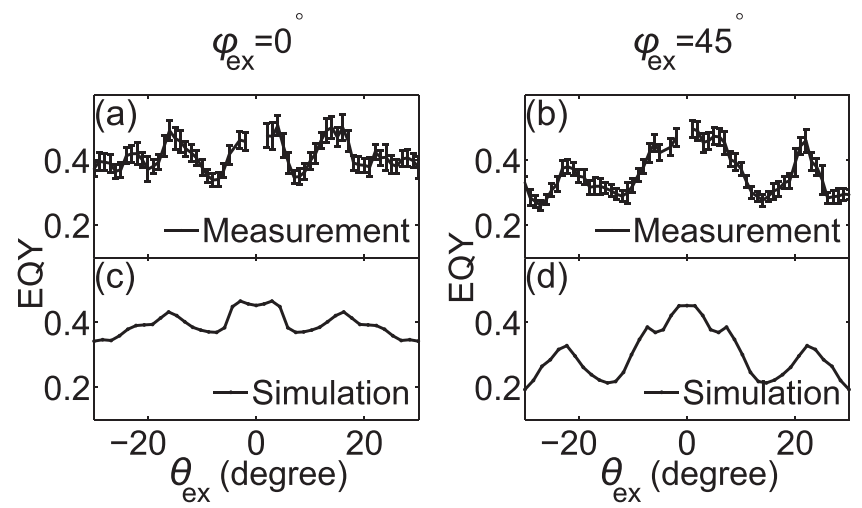

FIG. 6. (a) and (b) Measured and (c) and (d) simulated EQY as a function of $\theta_{\mathrm{ex}}$ for (a) and (c) $\varphi_{\mathrm{ex}}=0^{\circ}$ and (b) and (d) $\varphi_{\mathrm{ex}}=45^{\circ}$ for a square array of Al nanoparticles with a lattice constant of $400 \mathrm{~nm}$ covered by a $600 \mathrm{~nm}$ thick polymer layer doped with dye molecules.
EQY $\sim 0.3$ for $\varphi_{\mathrm{ex}}=45^{\circ}$. We attribute this reduction to the decrease in $\mathrm{A}_{\text {dye }}$ when the $\mathrm{A}_{\text {metal }}$ increases.

\section{CONCLUSIONS}

In conclusion, we have determined both experimentally and numerically the EQY of an optical antenna phased array consisting of an array of metallic ( $\mathrm{Al})$ nanoparticles and an emitting layer of dye molecules deposited on top. A custommade integrating sphere setup that enables variation of the angle of incidence, and numerical finite element simulations have been used to perform a detailed analysis of the EQY. Our results demonstrate a strong dependence of the EQY on the angle of incidence of the excitation beam, which has its origin in the distinct spatial distribution of the local electric field associated with the different modes supported by the nanoantenna phased array. These results demonstrate that the illumination conditions of plasmonic-based devices can lead to significant improvements of the EQY. Therefore, the illumination needs to be carefully designed and optimized in future experiments to achieve an optimum performance.

\section{ACKNOWLEDGMENTS}

We thank R. van Brakel, M. Lunz, S. R. K. Rodrìguez, D. K. G. de Boer, and D. J. Louwers for stimulating discussions. This work is part of the research program of the Foundation for Fundamental Research on Matter (FOM), which is financially supported by the Netherlands Organization for Fundamental Research (NWO). It is also part of an industrial partnership program between Philips and FOM. It is also supported in part by NanoNextNL, a micro and nanotechnology consortium of the Government of the Netherlands and 130 partners.

${ }^{1}$ P. Müuhlschlegel, H. J. Eisler, O. J. F. Martin, B. Hecht, and D. W. Pohl, "Resonant optical antennas," Science 308, 1607 (2005).

${ }^{2}$ G. A. Crosby and J. N. Demas, "Measurement of photoluminescence quantum yields. Review," J. Phys. Chem. 75, 991 (1971).

${ }^{3}$ C. Würth, M. Grabolle, J. Pauli, M. Spieles, and U. Resch-Genger, "Relative and absolute determination of fluorescence quantum yields of transparent samples," Nat. Protoc. 8, 1535 (2013).

${ }^{4}$ A. Kwadrin and A. F. Koenderink, "Gray-tone lithography implementation of Drexhage's method for calibrating radiative and nonradiative decay constants of fluorophores," J. Phys. Chem. C 116, 16666 (2012).

${ }^{5}$ P. Lunnemann, F. T. Rabouw, R. J. A. van Dijk-Moes, F. Pietra, D. Vanmaekelbergh, and A. F. Koenderink, "Calibrating and controlling the quantum efficiency distribution of inhomogeneously broadened quantum rods by using a mirror ball," ACS Nano 7, 5984 (2013).

${ }^{6}$ L. Porres, A. Holland, L.-O. Pålsson, A. P. Monkman, C. Kemp, and A. Beeby, "Absolute measurements of photoluminescence quantum yields of solutions using an integrating sphere," J. Fluoresc. 16, 267 (2006).

${ }^{7}$ D. O. Faulkner, J. J. McDowell, A. J. Price, D. D. Perovic, N. P. Kherani, and G. A. Ozin, "Measurement of absolute photoluminescence quantum yields using integrating spheres-Which way to go?," Laser Photonics Rev. 6, 802 (2012).

${ }^{8}$ J. R. Lakowicz, Principles of Fluorescence Spectroscopy (Springer, 2009). ${ }^{9}$ V. Giannini, J. A. Sánchez-Gil, O. L. Muskens, and J. Gómez Rivas, "Electrodynamic calculations of spontaneous emission coupled to metal nanostructures of arbitrary shape: Nanoantenna-enhanced fluorescence," J. Opt. Soc. Am. B 26, 1569 (2009).

${ }^{10} \mathrm{~J}$. Wenger, "Fluorescence enhancement factors on optical antennas: Enlarging the experimental values without changing the antenna design," Int. J. Opt. 2012, 828121 (2012). 
${ }^{11}$ S. Kühn, U. Håkanson, L. Rogobete, and V. Sandoghdar, "Enhancement of single-molecule fluorescence using a gold nanoparticle as an optical nanoantenna," Phys. Rev. Lett. 97, 017402 (2006).

${ }^{12} \mathrm{P}$. Anger, P. Bharadwaj, and L. Novotny, "Enhancement and quenching of single-molecule fluorescence,” Phys. Rev. Lett. 96, 113002 (2006).

${ }^{13}$ J. B. Khurgin, G. Sun, and R. A. Soref, "Enhancement of luminescence efficiency using surface plasmon polaritons: Figures of merit," J. Opt. Soc. Am. B 24, 1968 (2007).

${ }^{14}$ H. Mertens, A. F. Koenderink, and A. Polman, "Plasmon-enhanced luminescence near noble-metal nanospheres: Comparison of exact theory and an improved Gersten and Nitzan model," Phys. Rev. B 76, 115123 (2007).

${ }^{15} \mathrm{H}$. Mertens and A. Polman, "Strong luminescence quantum-efficiency enhancement near prolate metal nanoparticles: Dipolar versus higher-order modes," J. Appl. Phys. 105, 044302 (2009).

${ }^{16} \mathrm{C}$. P. Burrows and W. L. Barnes, "Large spectral extinction due to overlap of dipolar and quadrupolar plasmonic modes of metallic nanoparticles in arrays," Opt. Express 18, 3187 (2010).

${ }^{17}$ M. Liu, T.-W. Lee, S. K. Gray, P. Guyot-Sionnest, and M. Pelton, "Excitation of dark plasmons in metal nanoparticles by a localized emitter," Phys. Rev. Lett. 102, 107401 (2009).

${ }^{18}$ S. Zou, N. Janel, and G. C. Schatz, "Silver nanoparticle array structures that produce remarkably narrow plasmon lineshapes," J. Chem. Phys. 120, 10871 (2004).

${ }^{19}$ S. Zou and G. C. Schatz, "Silver nanoparticle array structures that produce giant enhancements in electromagnetic fields," Chem. Phys. Lett. 403, 62 (2005).

${ }^{20} \mathrm{G}$. Vecchi, V. Giannini, and J. Gómez Rivas, "Shaping the fluorescent emission by lattice resonances in plasmonic crystals of nanoantennas," Phys. Rev. Lett. 102, 146807 (2009).

${ }^{21}$ V. Giannini, G. Vecchi, and J. Gómez Rivas, "Lighting up multipolar surface plasmon polaritons by collective resonances in arrays of nanoantennas," Phys. Rev. Lett. 105, 266801 (2010).

${ }^{22}$ J. M. Steele, I. Gagnidze, and S. M. Wiele, "Efficient extraction of fluorescence emission utilizing multiple surface plasmon modes from gold wire gratings," Plasmonics 5, 319 (2010).

${ }^{23}$ G. Pellegrini, G. Mattei, and P. Mazzoldi, "Nanoantenna arrays for largearea emission enhancement," J. Chem. Phys. C 115, 24662 (2011).

${ }^{24}$ T. V. Teperik and A. Degiron, "Superradiant optical emitters coupled to an array of nanosize metallic antennas," Phys. Rev. Lett. 108, 147401 (2012).

${ }^{25}$ A. Abass, S. R. K. Rodriguez, T. Ako, T. Aubert, M. A. Verschuuren, D. van Thourhout, J. Beeckman, Z. Hens, J. Gómez Rivas, and B. Maes, "Active liquid crystal tuning of plasmonic enhanced light emission from colloidal quantum dots," Nano Lett. 14, 5555 (2014).

${ }^{26}$ K. H. Cho, J. Y. Kim, D.-G. Choi, K.-J. Lee, J.-H. Choi, and K. C. Choi, "Surface plasmon-waveguide hybrid polymer light-emitting devices using hexagonal Ag dots," Opt. Lett. 37, 761 (2012).

${ }^{27}$ G. Lozano, D. J. Louwers, S. R. K. Rodríguez, S. Murai, O. T. A. Jansen, M. A. Verschuuren, and J. Gómez Rivas, "Plasmonics for solid-state lighting: Enhanced excitation and directional emission of highly efficient light sources," Light: Sci. Appl. 2, e66 (2013).
${ }^{28}$ G. Lozano, G. Grzela, M. A. Verschuuren, M. Ramezani, and J. Gómez Rivas, "Tailor-made directional emission in nanoimprinted plasmonicbased light-emitting devices," Nanoscale 6, 9223 (2014).

${ }^{29}$ J. Stehr, J. Crewett, F. Schindler, R. Sperling, G. von Plessen, U. Lemmer, J. M. Lupton, T. A. Klar, J. Feldmann, A. W. Holleitner, M. Forster, and U. Scherf, "A low threshold polymer laser based on metallic nanoparticle gratings," Adv. Mater. 15, 1726 (2003).

${ }^{30}$ W. Zhou, M. Dridi, J. Y. Suh, C. H. Kim, D. T. Co, M. R. Wasielewski, G. C. Schatz, and T. W. Odom, "Lasing action in strongly coupled plasmonic nanocavity arrays," Nat. Nanotechnol. 8, 506 (2013).

${ }^{31}$ A. H. Schokker and A. F. Koenderink, "Lasing at the band edges of plasmonic lattices," Phys. Rev. B 90, 155452 (2014).

${ }^{32}$ S. Pillai, K. R. Catchpole, T. Trupke, and M. A. Green, "Surface plasmon enhanced silicon solar cells," J. Appl. Phys. 101, 093105 (2007).

${ }^{33}$ R. Adatoa, A. A. Yanika, J. J. Amsdenc, D. L. Kaplanc, F. G. Omenettoc, M. K. Honge, S. Erramillib, and H. Altuga, "Ultra-sensitive vibrational spectroscopy of protein monolayers with plasmonic nanoantenna arrays," Proc. Natl. Acad. Sci. U. S. A. 106, 19227 (2009).

${ }^{34}$ V. G. Kravets, F. Schedin, A. V. Kabashin, and A. N. Grigorenko, "Sensitivity of collective plasmon modes of gold nanoresonators to local environment," Opt. Lett. 35, 956 (2010).

${ }^{35}$ P. Offermans, M. C. Schaafsma, S. R. K. Rodriguez, Y. Zhang, M. CregoCalama, S. Brongersma, and J. Gómez Rivas, "Universal scaling of the figure of merit of plasmonic sensors," ACS Nano 5, 5151 (2011).

${ }^{36}$ M. A. Verschuuren, "Substrate conformal imprint lithography for nanophotonics," Ph.D. thesis, Utrecht University, Utrecht, 2010.

${ }^{37}$ S. R. K. Rodriguez, F. Bernal Arango, T. P. Steinbusch, M. A. Verschuuren, A. F. Koenderink, and J. Gómez Rivas, "Breaking the symmetry of forward-backward light emission with localized and collective magnetoelectric resonances in arrays of pyramid-shaped aluminum nanoparticles," Phys. Rev. Lett. 113, 247401 (2014).

${ }^{38}$ A. Christ, S. G. Tikhodeev, N. A. Gippius, J. Kuhl, and H. Giessen, "Waveguide-plasmon polaritons: Strong coupling of photonic and electronic resonances in a metallic photonic crystal slab," Phys. Rev. Lett. 91, 183901 (2003).

${ }^{39}$ S. Murai, M. A. Verschuuren, G. Lozano, G. Pirruccio, S. R. K. Rodriguez, and J. Gómez Rivas, "Hybrid plasmonic-photonic modes in diffractive arrays of nanoparticles coupled to light-emitting optical waveguides," Opt. Express 21, 4250 (2013).

${ }^{40}$ S. R. K. Rodriguez, S. Murai, M. A. Verschuuren, and J. Gómez Rivas, "Light-emitting waveguide-plasmon polaritons," Phys. Rev. Lett. 109, 166803 (2012).

${ }^{41}$ G. Lozano, T. Barten, G. Grzela, and J. Gómez Rivas, "Directional absorption by phased arrays of plasmonic nanoantennae probed with timereversed Fourier microscopy," New J. Phys. 16, 013040 (2014).

${ }^{42}$ M. Andreas Lieb, J. M. Zavislan, and L. Novotny, "Single-molecule orientations determined by direct emission pattern imaging," J. Opt. Soc. Am. B 21, 1210-1215 (2004).

${ }^{43}$ Comsol multiphysics ${ }^{\circledR}$, RF Module.

${ }^{44}$ E. D. Palik, Handbook of Optical Constants of Solids (Elsevier, 1998).

${ }^{45}$ M. Born and E. Wolf, Principle of Optics, 7 th ed. (Pergamon, 1999).

${ }^{46}$ E. Hutter and J. H. Fendler, "Exploitation of localized surface plasmon resonance," Adv. Mater. 16, 1685-1706 (2004). 